\title{
The Newborn of Diabetic Rat. II. Impaired Gluconeogenesis in the Postnatal Period
}

\author{
JOSE M. CUEZVA, ${ }^{(26)}$ CHELLAM I. CHITRA, ${ }^{(27)}$ AND MULCHAND S. PATEL ${ }^{(28)}$ \\ Departments of Biochemistry and Nutrition, Case Western Reserve University School of Medicine. Cleveland. Ohio, \\ $U S A$
}

Summary

The incorporation of $\left[\mathrm{U}-{ }^{14} \mathrm{C}\right]$ lactate into blood glucose, the alterations in the postnatal development of hepatic phospho-enolpyruvate ( $P$-enolpyruvate) carboxykinase activity, the concentrations of adenine nucleotides and the cytosolic $\mathrm{NAD}^{+} / \mathrm{NADH}$ ratios were measured in livers of the newborns of untreated and insulin-treated diabetic (streptozotocin induced) rats during the first $6 \mathrm{~h}$ of life. The rate of incorporation of $\left[{ }^{14} \mathrm{Cl}\right.$-lactate-carbon into glucose in the 6-h-old neonates of insulin-treated diabetic rats was about one-half of the rate observed in age-matched pups of normal rats. The rate of gluconeogenesis from labeled lactate in the 3-h-old neonates of untreated diabetic rats was approximately one-half of that observed in the age-matched pups of normal animals; however, the rates of gluconeogenesis in these two groups of neonates at $6 \mathrm{~h}$ of age were not significantly different. The levels of hepatic $P$-enolpyruvate carboxykinase activity were significantly lower in 3.5- and 6.5-h-old neonates of insulin-treated diabetic rats compared to the age-matched pups of normal rats whereas the activity of this enzyme was significantly lower only in livers of 0.5 - and 3.5-h-old neonates of untreated diabetic rats compared to the age-matched pups of normal animals.

At birth and during the first 3 postnatal $h$, the concentrations of hepatic ATP and the adenylate energy charge were significantly higher in the newborns of insulin-treated diabetic rats compared to both normal and untreated diabetic rats; however, a significant reduction in these parameters was observed after 3 postnatal $h$ in neonates of insulin-treated diabetic rats compared to both normal and untreated diabetic animals. The postnatal increase in the hepatic cytosolic redox state $\left(\mathrm{NAD}^{+} / \mathrm{NADH}\right)$ in the newborns of insulin-treated diabetic rats was similar to that observed in the newborn pups of normal animals during the first $3 \mathrm{~h}$ postnatal period; however, a significant decline in this parameter was observed only in the neonates of insulin-treated diabetic rats after the third postnatal $h$. The postnatal increase in the hepatic cytosolic redox state $\left(\mathrm{NAD}^{+} / \mathrm{NADH}\right)$ was significantly lower in the neonates of untreated diabetic rats compared to the age-matched pups of normal rats during the entire $9 \mathrm{~h}$ experimental period.

The time course of the postnatal changes in the induction of Penolpyruvate carboxykinase activity, the concentrations of adenine nucleotides and the cytosolic redox state in livers of the newborn rats shows that attenuated postnatal development of hepatic $P$ enolpyruvate carboxykinase and the energy state are largely responsible for observed impaired gluconeogenesis from lactate in the neonates of insulin-treated diabetic rats.

\section{Speculation}

Postnatal alterations in the concentrations of adenine nucleotides and the cytosolic redox state in livers of the neonates of insulin-treated diabetic rats suggest an impaired hepatic mitochondrial metabolism in the immediate postnatal period. Studies of the uptake or transport of adenine nucleotides and anions by isolated mitochondria from livers of the neonates of insulin-treated diabetic rats would aid in our understanding of these changes, and possibly would lead to identification of factor(s) responsible for these changes in livers of newborns of diabetic mothers.

In the human, newborn infants of uncontrolled or poorly controlled diabetic mothers often experience severe hypoglycemia (8, 17). Fetal hyperinsulinemia caused by hyperglycemia in utero enhances the disappearance of glucose from the circulation in the newborns of diabetic mothers in the immediate postnatal period (17). Furthermore, it is observed that the newborn infants of insulin-dependent diabetic mothers release glucose in the blood stream at a lower rate than the infants of normal mothers (15). The contribution of hepatic gluconeogenesis to the total glucose production in the newborns of diabetic mothers is, however, not known. In the preceding paper (9), we demonstrated that the newborns of insulin-treated diabetic rats had several clinical features such as macrosomia, hyperinsulinemia, prolonged hypoglycemia and impaired glucagon secretory response, similar to those observed in the newborn infants of insulin-dependent diabetic women. In the same study (9), we also observed a delay in the initiation of hepatic glycogenolysis in the newborns of diabetic rats compared to normal animals. In the present study, we report the alterations in the rate of gluconeogenesis and the development of hepatic cytosolic phosphoenolpyruvate carboxykinase in newborns of both untreated and insulin-treated diabetic rats during the first 6 postnatal $\mathrm{h}$. The possible influence on hepatic gluconeogenesis by the postnatal changes in the energy state and the cytosolic redox state in livers of newborns of diabetic rats was also investigated.

\section{MATERIALS AND METHODS}

Animals. Timed pregnant (overnight mating) Sprague-Dawley rats (25) weighing $200 \mathrm{~g}$ were fed Purina Laboratory chow and water ad libitum. Diabetes mellitus was induced by injecting streptozotocin $(45 \mathrm{mg} / \mathrm{kg}$ body weight) in $50 \mathrm{mM}$ sodium citrate

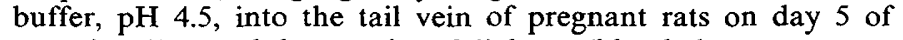
gestation (11), and the severity of diabetes (blood glucose concentrations of $15 \mathrm{mM}$ or higher from day 7 to 22 of gestation) was monitored as described in the preceding paper (9). Insulin-treated diabetic (mildly hyperglycemic) pregnant rats were obtained from the group of severely diabetic mothers; their hyperglycemia was controlled with two daily subcutaneous injections of insulin (NPH, U-100) $1-2$ units at $0900 \mathrm{~h}$ and 2-4 units at $1700 \mathrm{~h}$ from day 18 of pregnancy until delivery (9). The insulin dose injected was determined by measuring the tail vein blood glucose concentration (6) immediately before the injection of insulin. The regimen of insulin administration reduced the blood glucose concentration from severely hyperglycemic ( $270 \mathrm{mg}$ of glucose/dl or higher) to mildly hyperglycemic (80-200 $\mathrm{mg}$ of glucose/dl) levels. The details of this treatment are reported in the preceding paper (9). Term fetuses (22-day-old) were delivered by cesarian section after cervical dislocation of the mother. Newborns were quickly detached 
from the placentas after tying the umbilical cords and maintained at $37^{\circ} \mathrm{C}$ in a humidicrib without feeding as described in the preceding paper (9). No sign of hypoxia was observed in these neonates during the experimental period.

In vivo studies. Twenty $\mu \mathrm{l}$ of $0.9 \% \mathrm{NaCl}$ containing $1.3 \mu \mathrm{Ci}$ of $\mathrm{L}$ $\left[\mathrm{U}-{ }^{14} \mathrm{C}\right]$-lactate were injected intraperitoneally into the neonates at 3 or $6 \mathrm{~h}$ after delivery, and the pups were decapitated and 100 microliters of blood collected at the specific time intervals. Aliquots of neutralized samples were chromatographed on Dowex 1$\mathrm{X} 8$ chloride column to separate glucose and lactate (1). The radioactivity (1) as well as the concentrations of glucose (6) and lactate (12) were measured in these fractions. To measure the incorporation of radioactivity into glycogen, livers were digested in $30 \% \mathrm{KOH}$, and glycogen was isolated and washed four times. The concentration of glycogen was determined by the method of Van Handel (20).

The activity of phosphoenolpyruvate (P-enolpyruvate) carboxykinase was measured in liver cytosol (5). One unit of activity represents $1 \mu$ mole of $\mathrm{H}^{14} \mathrm{CO}_{3}$ fixed $/ \mathrm{min}$ at $37^{\circ} \mathrm{C}$.

Freeze-clamped studies. The livers of the first two fetuses, which were still attached to the mother, were frozen immediately using Wollenberger clamps precooled in liquid $\mathrm{N}_{2}$ (24). These samples obtained within $30 \mathrm{sec}$ after cervical dislocation of the mother were considered the zero time in all experiments. At various time intervals after delivery the livers of the neonates were freezeclamped before decapitation of the pups. Frozen livers were pulverized in a precooled mortar and extracted with $6 \%$ perchloric acid. ATP (16), ADP (14), AMP (14), pyruvate (14), and lactate (12) were measured in the neutralized extracts using standard enzymatic procedures. Using the metabolite levels, the adenylate energy charge (4) and the cytosolic redox state $\left(\mathrm{NAD}^{+} / \mathrm{NADH}\right)$ (23) of the livers were calculated. cAMP was measured in the perchloric acid-ethanol extract of the freeze-clamped liver using a cAMP binding protein (7). Statistical analysis was performed using the Student's $t$ test for non-paired samples, and a comparison was made with the control group. $P$ values of 0.05 or less were taken as significant, and the results are expressed as the mean \pm S.E. In both in vivo and freeze-clamped studies, two pups from the same litter were allotted to each experimental period; occasionally. when only one pup from the litter was assigned per a given time period, more mothers were used in these experiments to obtain the necessary number of observations per experimental period as indicated in the legends to figures.

\section{RESULTS}

Incorporation of ${ }^{14} \mathrm{CJ}$-lactate-carbon into blood glucose and hepatic glycogen. During the two experimental periods (at 3 and 6 postnatal $h$ ) the concentrations of lactate in blood of three groups of neonates remained in a steady state (Fig. 1). Similarly, the concentrations of blood glucose in the three groups of neonates also remained in a steady state over a period of at least $30 \mathrm{~min}$ after the administration of labeled lactate (results not shown). The changes in the specific radioactivities of lactate and glucose in the blood from 3-h and 6-h-old neonates of normal, untreated and insulin-treated diabetic rats are depicted in Figure 1. The specific radioactivity of lactate (at $t=0$ ) calculated from the plot of $\log$ specific radioactivity versus time was similar (35000-38000 dpm/ $\mu$ mole) in the three groups of neonates. Earlier it was demonstrated that the decay curve of the specific radioactivity of plasma lactate in newborn rats after injection of the labeled substrate followed a binary pattern with a fast and a slow component (21). To avoid previously observed high values for the lactate body pool size and lactate space higher than $100 \mathrm{ml} / 100 \mathrm{~g}$ body weight $(21)$ and because the experimental data fitted into the slow component of the decay curve, we have expressed our results as d.p.m. incorporated into $\mu$ mole of blood glucose $/ \mathrm{min} / 100 \mathrm{~g}$ body weight (Fig. 2 ). Three $h$ after birth the incorporation of $\left[{ }^{14} \mathrm{C}\right]$ carbon from lactate into glucose in the neonates of normal and insulin-treated diabetic rats was similar but approximately 2 -fold higher than that observed in the age-matched neonates of untreated diabetic rats

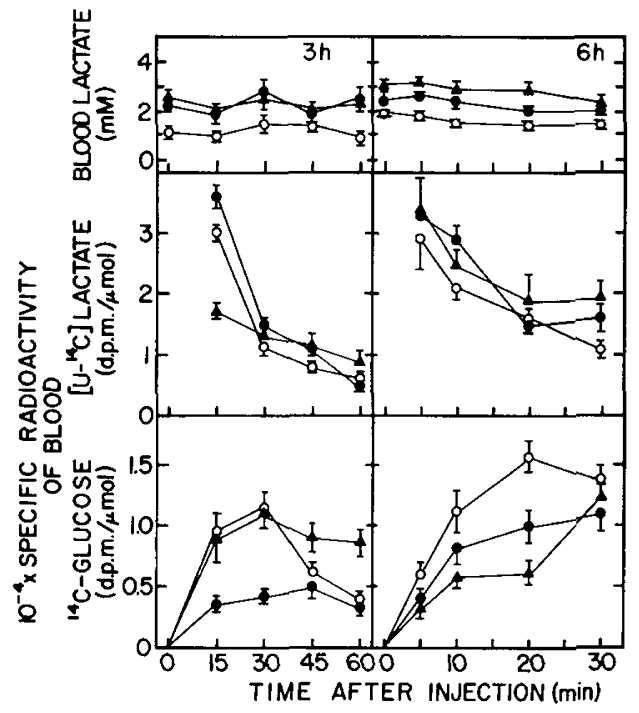

Fig. 1. Changes in the specific radioactivities of blood lactate and glucose after the injection of $\mathrm{L}-\left[\mathrm{U}^{-14} \mathrm{C}\right]$ lactate in 3- and 6-h-old newborns of normal $(O)$, untreated diabetic $(\boldsymbol{O})$ and insulin-treated diabetic $(\boldsymbol{\Delta})$ rats. Newborns were maintained at $37^{\circ} \mathrm{C}$ in a humidicrib and without feeding. At 3 or $6 \mathrm{~h}$ after Cesarian delivery, each pup was injected with $1.3 \mu \mathrm{Ci}$ of $\mathrm{L}-\left[\mathrm{U}-{ }^{14} \mathrm{C}\right]$-lactate. After indicated time intervals the pups were killed and the radioactivity in blood lactate and glucose was measured. The results are the means $\pm S$.E. for six to twelve neonates from three to six rats.

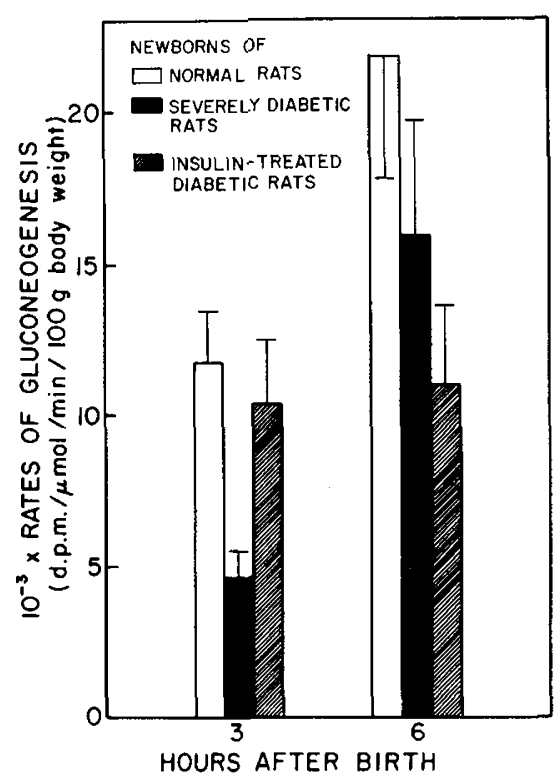

Fig. 2. The rate of incorporation of $\left[\mathrm{U}^{14} \mathrm{C}\right]$ lactate-carbon into blood glucose in 3- and 6-h-old newborns of normal, untreated and insulintreated diabetic rats. These rates are calculated from the data shown in Figure 1. The results are expressed as d.p.m./ mole of blood glucose $/ \mathrm{min} /$ $100 \mathrm{~g}$ body weight, and are the means \pm S.E. for six to twelve neonates from three to six rats.

(Fig. 2). Six h after birth the rate of gluconeogenesis from labeled lactate in the neonates of normal rats was increased 2-fold compared with the $3-\mathrm{h}$ period. A 3 -fold increase in the rate of gluconeogenesis was observed in the neonates of untreated diabetic rats between 3 and $6 \mathrm{~h}$ after birth (Fig. 2), and the rate of gluconeogenesis in this group of pups at the sixth $h$ was not significantly different from that observed in age-matched normal pups. In contrast, the neonates of insulin-treated diabetic rats, which had similar rates of gluconeogenesis compared to the normal pups at the third postnatal $h$, had only about one-half of 
the rate observed in age-matched 6-h-old normal pups. When the data were calculated as d.p.m. in glucose $\times 10^{-3} / \mathrm{ml}$ of blood/ $\mathrm{min} / 100 \mathrm{~g}$ body weight (at $3 \mathrm{~h}: 52.4 \pm 7.8,38.5 \pm 7.7$ and $11.4 \pm$ 2.0 and at $6 \mathrm{~h}: 108.0 \pm 18.0,42.9 \pm 9.9$ and $103.5 \pm 24.5$ for neonates of normal, untreated and insulin-treated diabetic mothers, respectively), the findings were essentially similar to that described in Figure 2. Similarly, when the data were calculated as $\mu$ mole of lactate incorporated into glucose/min/pup, the differences in the rate of gluconeogenesis among the three groups of neonates were also similar to those depicted in Figure 2. The incorporation of $\left[{ }^{1.4} \mathrm{C}\right]$ carbon into glycogen was negligible and no differences were observed between the three groups of neonates studied at both time periods (results not shown).

Induction of P-enolpyruvate carboxykinase. Figure 3 depicts the induction of the cytosolic P-enolpyruvate carboxykinase activity in livers of the newborns of normal, insulin-treated and untreated diabetic rats during the first $6.5 \mathrm{~h}$ after birth. The developmental pattern of this enzyme in livers of normal neonates was similar to that reported previously $(5,13)$. The activity of P-enolpyruvate carboxykinase in livers of neonates of untreated diabetic rats at 0.5 and $3.5 \mathrm{~h}$ was significantly lower compared to age-matched neonates of normal mothers; however, there was no significant difference in the levels of activity between these two groups of neonates at $6.5 \mathrm{~h}$ of age (Fig. 3; also see insert). The levels of activity in livers of 3.5- and 6.5-h-old neonates of insulin-treated diabetic animals were significantly lower $(P<0.001)$ than those observed in the age-matched pups of normal rats. Because the postnatal induction of the cytosolic P-enolpyruvate carboxykinase is modulated by hepatic cAMP concentrations $(13,22)$, we measured the concentrations of cAMP in three groups of neonates. As reported in the preceding paper (9), the concentrations of hepatic cAMP in the newborns of normal rats increased approximately 1.5-fold during the first 2 postnatal $h$. In contrast, there was no significant increase in the concentrations of hepatic cAMP in the newborns of untreated and insulin-treated diabetic rats during the first 2 postnatal h; however, during the third postnatal $h$ a significant increase in hepatic cAMP concentration was observed in the neonates of both insulin-treated $(P<0.01)$ and untreated $(P<$ $0.001)$ diabetic rats compared to their respective zero $h$ values (9). At 3 and 6 postnatal $h$, there was no significant difference in the concentrations of hepatic CAMP among three groups of neonates.

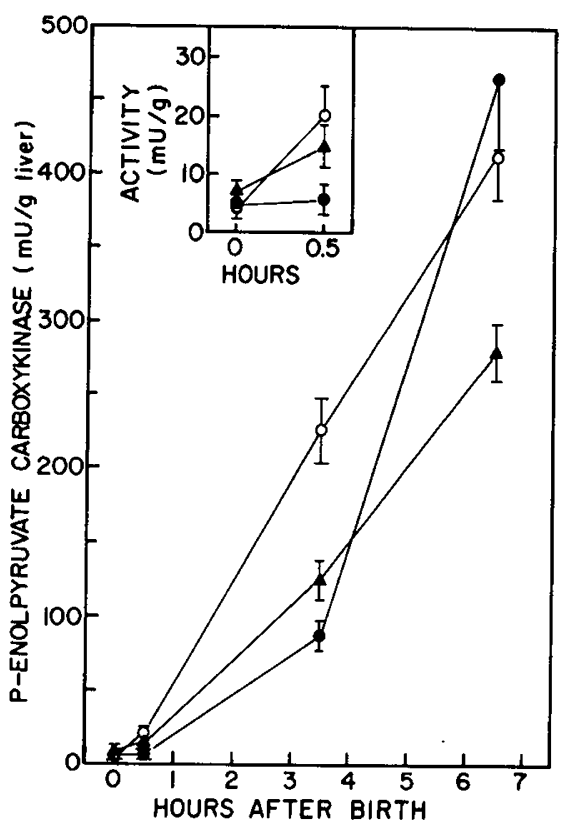

Fig. 3. Hepatic cytosolic phosphoenolpyruvate carboxykinase activity in the newborns of normal $(\bigcirc)$, untreated diabetic $(\bullet)$, and insulin-treated diabetic $(\boldsymbol{\Delta})$ rats during the first 6.5 postnatal $h$. The results are the means \pm S.E. for six to twenty-four neonates from three to twelve rats.

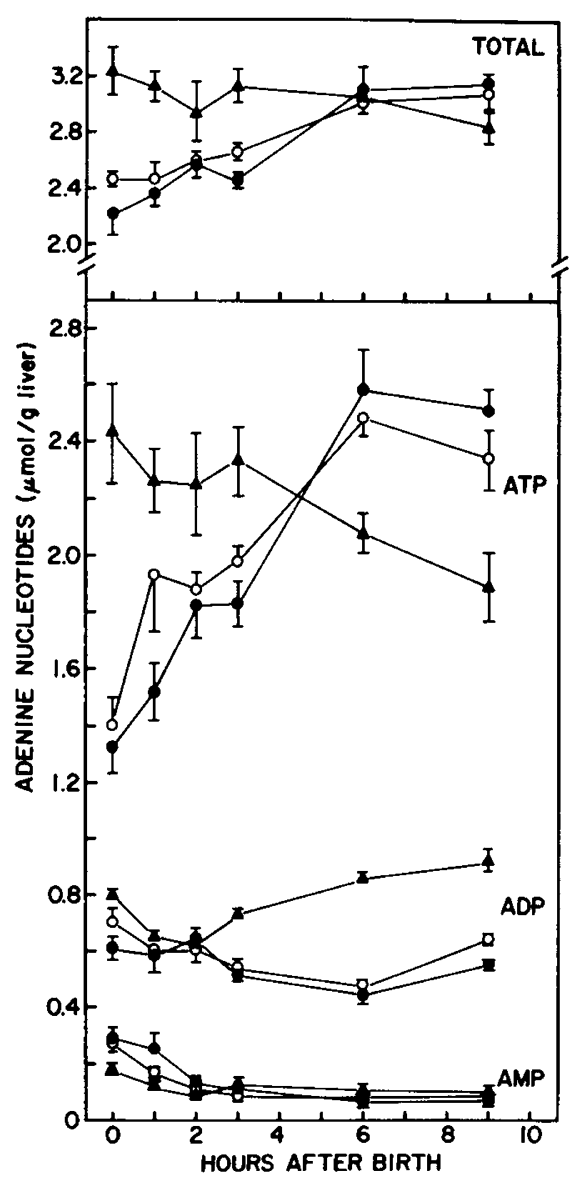

Fig. 4. Liver adenine nucleotides concentrations in the newborns of normal $(O)$, untreated diabetic $(\Theta)$, and insulin-treated diabetic $(\boldsymbol{\Delta})$ rats during the first 9 postnatal $h$. The results are the means \pm S.E. for six to twelve neonates from six to twelve rats.

Changes in the concentrations of adenine nucleotides, pyruvate and lactate in the liver. At the time of delivery, hepatic concentrations of ATP, ADP and AMP in the newborns of normal and untreated diabetic rats were not significantly different, whereas the concentrations of hepatic ATP and ADP were significantly higher $(P<0.001)$ and AMP concentrations significantly lower $(P<0.01)$ in the newborns of insulin-treated diabetic rats compared to normal animals (Fig. 4). This resulted in a higher concentration of total adenine nucleotides in the livers of insulintreated diabetic rats compared to normal animals (Fig. 4). The concentrations of ATP in livers of the newborns of normal and untreated diabetic rats increased markedly during the first 2 postnatal $h$ whereas no significant change was observed in ATP concentrations in the newborns of insulin-treated diabetic rats during this period. A second postnatal increase of a similar magnitude in the concentrations of ATP in livers of newborns of both normal and untreated diabetic rats was observed from 3-6 postnatal $h$. In contrast, a significant decrease in the concentrations of hepatic ATP was observed in the neonates of insulin-treated diabetic rats at the sixth and ninth postnatal $h$ compared to its third $h$ value and to the concentrations in age-matched newborns of normal animals (Fig. 4). The decrease in the concentrations of hepatic ATP in the neonates of insulin-treated diabetic rats was accompanied by an increase in the concentrations of ADP with no significant changes in the total adenine nucleotide concentrations in this group of neonates. In contrast, a significant increase $(P<0.005)$ in the total adenine nucleotide concentrations was observed in the neonates of normal and untreated diabetic rats during the same experimental period.

The adenylate energy charge (ATP + 1/2 ADP)/(ATP + ADP + AMP) (4) in livers of the newborns of normal and untreated 
diabetic rats were approximately 0.74 at birth and steadily increased to 0.9 in both groups of pups by the sixth postnatal h (Fig. 5 ). In contrast, the adenylate energy charge in the newborns of insulin-treated diabetic rats was 0.84 and remained unchanged until the third postnatal $h$ at which time no significant difference was observed among the three groups of neonates. During the next $6 \mathrm{~h}$, a significant reduction in the adenylate energy charge was observed in the neonates of insulin-treated diabetic rats, and these values were markedly lower than comparable values in the neonates of normal rats (Fig. 5). When these results are expressed as the ATP/ADP ratio during the first 6 postnatal $h$, a steady increase in the ATP/ADP ratio (from 2.05 at birth to 5.1 at $6 \mathrm{~h}$ ) was observed in the neonates of normal rats (Fig. 5). After a delay of $2 \mathrm{~h}$, the ATP/ADP ratio increased markedly (from 2.35 at $2 \mathrm{~h}$ to 5.9 at $6 \mathrm{~h}$ ) in the neonates of untreated diabetic rats. A significant reduction in the ATP/ADP ratios was, however, observed in the pups of both normal and untreated diabetic rats at the ninth postnatal $h$. The ATP/ADP ratio in livers of neonates of insulin-treated diabetic rats varied only slightly during the first 3 postnatal h, however, it declined steadily over the next $6 \mathrm{~h}$ (from 3.0 at three h to 1.95 at nine h) (Fig. 5). The ATP/ADP ratios in the pups of insulin-treated diabetic rats at both 6 and 9 postnatal $\mathrm{h}$ were markedly lower than the corresponding ratios in the pups of normal rats.

Within $1 \mathrm{~h}$ after birth a significant increase in the hepatic pyruvate concentration was observed in all three groups of newborns (Fig. 6). After the first postnatal h, the concentrations of pyruvate in livers of the three groups of neonates declined significantly, but hepatic pyruvate concentrations in pups of untreated diabetic rats remained significantly higher than those in agematched neonates of normal and insulin-treated diabetic rats (Fig. 6 ). At birth the concentrations of lactate in livers of newborns of untreated diabetic rats were significantly $(P<0.05)$ higher than those in the newborns of normal rats. During the first $h$ of extrauterine life, the concentration of lactate in livers of neonates of untreated diabetic rats increased 2 -fold whereas no changes were observed in hepatic lactate concentrations in age-matched pups of both normal and insulin-treated diabetic rats, and these differences were maintained during the next $2 \mathrm{~h}$. The calculated

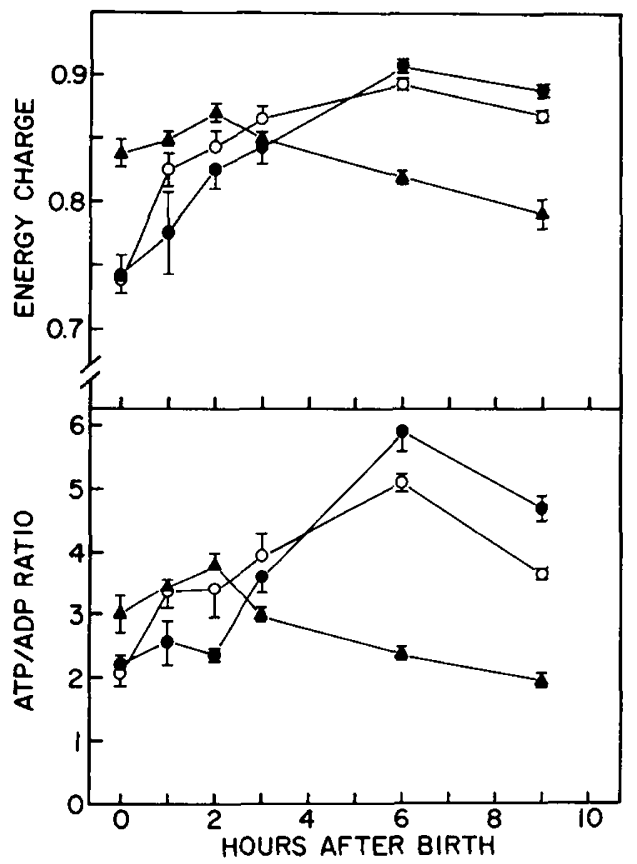

Fig. 5. Postnatal changes in the adenylate energy charge and ATP/ ADP ratio in livers of newborns of normal $(O)$, untreated diabetic $(\bullet)$. and insulin-treated diabetic $(\mathbf{\Delta})$ rats during the first 9 postnatal $h$. These data are calculated from the results shown in Figure 4. The results are the means \pm S.E. for six to twelve neonates from six to twelve rats.

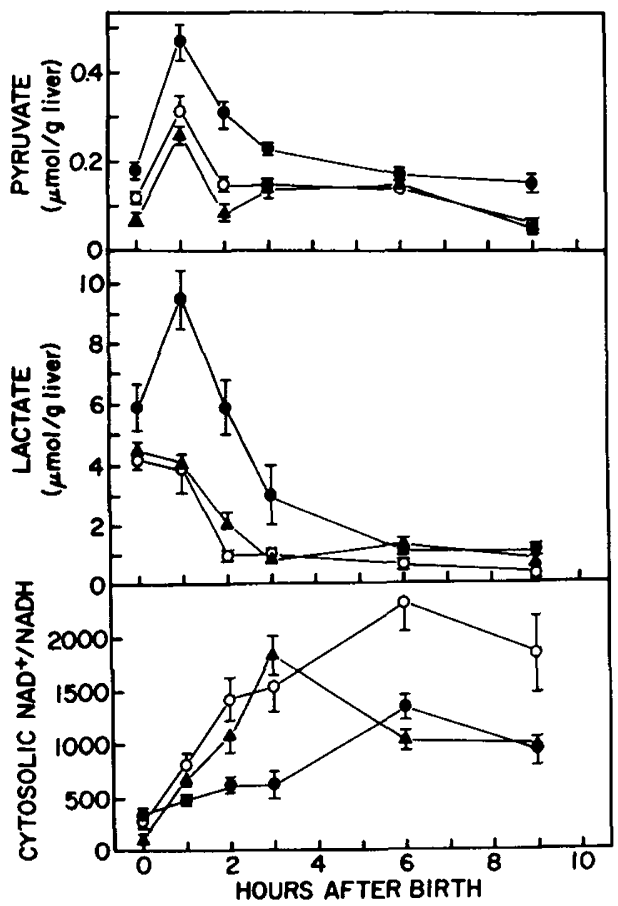

Fig. 6. Liver pyruvate and lactate concentrations and the calculated cytosolic $\mathrm{NAD}^{+} / \mathrm{NADH}$ ratios in livers of newborns of normal $(O)$, untreated diabetic $(\boldsymbol{O})$, and insulin-treated diabetic $(\boldsymbol{A})$ rats during the first 9 postnatal $h$. The results are the means \pm S.E. for six to twelve neonates from six to twelve rats.

cytosolic $\mathrm{NAD}^{+} / \mathrm{NADH}$ ratio (23) in livers of newborns of normal rats increased steadily reaching a maximum level at $6 \mathrm{~h}$ after birth (Fig. 6). In the neonates of untreated diabetic rats, the hepatic cytosolic $\mathrm{NAD}^{+} / \mathrm{NADH}$ ratio did not change significantly from birth to $3 \mathrm{~h}$, but increased significantly during the next three $\mathrm{h}$; however, the hepatic cytosolic $\mathrm{NAD}^{+} / \mathrm{NADH}$ ratios in this group of neonates were significantly lower than those in the age-matched pups of normal rats at all time periods except at birth. The increase in the hepatic cytosolic redox state $\left(\mathrm{NAD}^{+} / \mathrm{NADH}\right)$ in the newborns of insulin-treated diabetic rats during the first 3 postnatal $h$ was similar to that observed in the newborns of normal rats. During the next $6 \mathrm{~h}$, the hepatic cytosolic NAD ${ }^{+} / \mathrm{NADH}$ ratio in the neonates of insulin-treated diabetic rats declined significantly reaching the values observed in age-matched pups of untreated diabetic rats.

\section{DISCUSSION}

The results of this study show alterations in the rates of gluconeogenesis from lactate in the newborns of diabetic rats during the first 6 postnatal $h$. These changes will be discussed in light of alterations in (1) the postnatal emergence of hepatic cytosolic Penolpyruvate carboxykinase activity and (2) the energy state and the cytosolic redox state in the livers of neonates of diabetic rats.

Alterations in the postnatal induction of hepatic cytosolic P-enolpyruvate carboxvkinase and gluconeogenesis in the newborns of diabetic rats. Previous studies have conclusively demonstrated that the synthesis of cytosolic P-enolpyruvate carboxykinase in livers of the term fetuses (premature induction) and newborns of normal rats (postnatal physiologic induction) is a cAMP-dependent process (13). The emergence of P-enolpyruvate carboxykinase in livers of newborns of normal rats within the first few $h$ after birth is caused by a chain of events leading to an increase in the hepatic cAMP concentrations. In the present study the activity of hepatic P-enolpyruvate carboxykinase was significantly lower in 3.5 -h-old neonates of both untreated and insulin-treated diabetic rats compared to age-matched normal pups (Fig. 3). This is consistent with the observed delay in the increase in concentrations of hepatic 
cAMP in the newborns of diabetic rats during the first 2 postnatal h (9), and with the relative hyperinsulinemia (2-3-fold higher) maintained by these two groups of neonates compared to agematched pups of normal rats (9). The administration of exogenous insulin to the term fetuses (22) and newborn rats (10) is shown to cause a reduction in the cAMP-dependent induction of hepatic $\mathrm{P}$ enolpyruvate carboxykinase. Using cultured rat fetal hepatocytes, Steele et al. (18) recently demonstrated that insulin partially inhibited the induction of this enzyme by $\mathrm{Bt}_{2} \mathrm{cAMP}$. Although the concentrations of hepatic cAMP in the three groups of neonates were not significantly different from 3-6 postnatal $h$, the activity of P-enolpyruvate carboxykinase was still significantly lower in 6.5 -h-old neonates of insulin-treated diabetic rats compared to age-matched pups of normal and untreated diabetic rats. In this case it is possible that a reduced energy state in livers of the neonates of insulin-treated diabetic rats compared to normal rats during the period from 3-6 postnatal h (Fig. 4) may have contributed to low levels of enzyme activity in 6.5 -h-old pups of insulintreated diabetic rats.

The data show that the rates of incorporation of lactate-carbon into glucose in the neonates of diabetic rats at 3 and $6 \mathrm{~h}$ after birth were largely determined by the activity of hepatic P-enolpyruvate carboxykinase, the energy state and the cytosolic redox state in the liver as demonstrated earlier in the newborns of normal rats by Ballard (3). Despite having a significantly lower level of hepatic P-enolpyruvate carboxykinase activity in 3-h-old neonates of insulin-treated diabetic rats compared to age-matched pups of normal rats, the rates of $\left[{ }^{14} \mathrm{C}\right]$ lactate incorporation into blood glucose were not significantly different between these two groups of pups. The energy state and the cytosolic $\mathrm{NAD}^{+} / \mathrm{NADH}$ ratio were similar in both groups of pups at this age; however, the rate of gluconeogenesis from lactate was significantly lower in the 3-h-old pups of untreated diabetic rats, which had a significantly lower level of hepatic P-enolpyruvate carboxykinase activity and a very low cytosolic $\mathrm{NAD}^{+} / \mathrm{NADH}$ ratio in the liver compared to age-matched neonates of normal animals (Fig. 6). Similarly, a significant reduction in the rate of gluconeogenesis was observed in 6-h-old neonates of insulin-treated diabetic animals, which also had a very low energy state and the cytosolic redox state in addition to low activity of P-enolpyruvate carboxykinase in the liver.

Alterations in the postnatal development in the energy state and the cytosolic $N A D^{+} / N A D H$ ratio in livers of diabetic rats. The postnatal changes in the concentrations of adenine nucleotides, the adenylate energy charge and the cytosolic $\mathrm{NAD}^{+} / \mathrm{NADH}$ ratio in livers of newborns of normal rats as described in this study are similar to those reported previously (3). Surprisingly, the newborns of insulin-treated diabetic rats had a higher (total) and highly phosphorylated (ATP) pool of hepatic adenine nucleotides at birth and during the first 3 postnatal $h$ than those observed in the age-matched newborns of normal rats (Fig. 4). The reason for such a change is not clear; however, an enhanced glycolysis and/ or early maturation of mitochondrial oxidative metabolism (see below) in the term fetuses of insulin-treated diabetic rats compared to normal animals could cause such changes. The reduction in the hepatic ATP concentrations in the neonates of insulin treated diabetic rats after the third postnatal $h$ is also difficult to explain; however, this reduction was not caused by prolonged lowered blood glucose observed in these pups during the first 3 postnatal $h$ because ( 1 ) the reduction in ATP concentrations was observed when these pups were euglycemic after 3 postnatal $h$ and (2) the administration of exogenous glucose to these newborns ( 110 $\mu$ moles of glucose at birth and also at $3 \mathrm{~h}$ of life) did not alter the postnatal changes in the concentrations of hepatic adenine nucleotides. [Hepatic ATP concentrations ( $\mu$ moles/g liver) at 1,3 and $6 \mathrm{~h}$ after saline or glucose injection into the newborns at birth and also at $3 \mathrm{~h}$ of life were as follows. Pups of normal mothers: saline injected, $2.00 \pm 0.23$ at $\mathrm{l} \mathrm{h}$ and $2.12 \pm 0.09$ at $3 \mathrm{~h}$; glucose injected, $1.42 \pm 0.14(P<0.05)$ at $1 \mathrm{~h}$ and $1.82 \pm 0.29$ at $3 \mathrm{~h}$. Pups of insulin-treated mothers: saline injected, $2.40 \pm 0.03$ at $1 \mathrm{~h}, 2.39$ \pm 0.07 at $3 \mathrm{~h}$ and $2.03 \pm 0.08$ at $6 \mathrm{~h}$; glucose injected, $2.55 \pm 0.05$ at $1 \mathrm{~h}, 2.29 \pm 0.06$ at $3 \mathrm{~h}$ and $2.07 \pm 0.05$ at $6 \mathrm{~h} ; n=8-10$ neonates from four to five mothers].

It has been proposed that the maturation of mitochondria (as judged by an increase in the level as well as the transport of mitochondrial adenine nucleotides and in the respiratory control index) in rat liver occurs soon after birth $(2,19)$. It is argued that the increase in the cytosolic ATP concentration, which is responsible for the initiation of the mitochondrial maturation, is brought about by a chain of reactions involving the secretion of glucagon causing an increase in hepatic cAMP concentrations which in turn enhances the rates of hepatic glycogenolysis and glycolysis (19). Although the postnatal increase in the plasma glucagon concentration was absent in the newborns of both untreated and insulintreated diabetic rats compared to normal animals during the first $2 \mathrm{~h}$ of extrauterine life, there was an increase in hepatic ATP levels in the 2-h-old newborns of untreated diabetic rats, whereas the concentrations of ATP in livers of newborns of insulin-treated diabetic rats were already elevated at birth. Our findings indicate that the postnatal increase in the hepatic ATP concentrations in newborn rats during the first 2 postnatal h may not depend upon the increase in the concentrations of plasma glucagon and hepatic cAMP as suggested by Sutton and Pollak (19). The data also show that in spite of the high hepatic ATP concentrations in the newborns of insulin-treated diabetic rats at birth and during the first 3 postnatal h, there were alterations in hepatic mitochondrial metabolism as evidenced by a decline in the total ATP concentrations and hepatic cytosolic $\mathrm{NAD}^{+} / \mathrm{NADH}$ ratios in this group of neonates after the third postnatal $\mathrm{h}$. Our findings suggest that factor(s) other than the ATP concentration may influence the postnatal changes in the mitochondrial metabolism in rat liver, and the newborns of insulin-treated diabetic rats may prove useful in identifying the mechanism(s) responsible for alterations in these parameters in the liver.

In summary, prolonged hypoglycemia observed in the newborns of insulin-treated diabetic rats during the postnatal period is caused by specific alterations in carbohydrate metabolism. In the preceding paper (9), we documented that postnatal hyperinsulinemia, attenuated glucagon secretion response and a delayed increase in the concentration of hepatic cAMP in the newborns of insulin-treated and untreated diabetic rats caused a delay in the initiation of hepatic glycogenolysis in these two groups of neonates. The results presented here show that there is also a delay in the induction of cytosolic P-enolpyruvate carboxykinase in the neonates of insulin-treated and untreated diabetic rats. Evidence is also presented to show that in addition to the reduced level of enzyme activity, the alterations in the energy state and the cytosolic redox state in the liver contributed significantly to low rates of gluconeogenesis observed in the neonates of insulin-treated diabetic rats.

\section{REFERENCES AND NOTES}

1. Adam, P. A. J.. Raiha, N. Rahiala. E.-L., and Kekomaki, M.: Oxidation of glucose and D-B-OH-butyrate by the early human fetal brain. Acta Paediatr. Scand., 64: 17 (1975)

2. Aprille. J. R. and Asikamis, G. K.: Postnatal development of rat mitochondria: state 3 respiration. adenine nucleotide translocase activity, and the net accumulation of adenine nucleotides. Arch. Biochem. Biophys.. 201: 564 (1980).

3. Ballard. F. J.: The development of gluconeogenesis in rat liver. Controlling factors in the newborn. Biochem. J.. 124: 265 (1971).

4. Ballard. F. J.: Adenine nucleotides and the adenylate kinase equilibrium in livers of foetal and newborn rats. Biochem. J., 117: 231 (1970).

5. Ballard. F. J. and Hanson, R. W.: Phosphoenolpyruvate carboxykinase and pyruvate carboxylase in developing rat liver. Biochem. J.. 104: 866 (1967)

6. Bergmeyer. H. V., Bernt. E., Schmidt, F., and Stork, H.: D-glucose determination with hexokinase and glucose-6-phosphate dehydrogenase. In: H. V. Bergmeyer: Methods in Enzlmatic Analysis. Vol. 3. pp. 1196-1201 (Academic Press. New York. 1974).

7. Brown, B. L., Albano, J. D. M., Ekins. R. P., Sgherzi, A. M. and Tampion. W. A simple and sensitive saturation assay method for the measurement of adenosine 3':5'-cyclic monophosphate. Biochem. J., 121:561 (1971).

8. Cornblath. M. and Schwartz, R.: Disorders of carbohydrate metabolism in infancy. Major problems in clinical pediatrics. Vol. 3, pp. 57-81 (W. B. Saunders. Philadelphia. 1966).

9. Cuezva. J. M., Burkett. E. S., Kerr, D. S., Rodman, H. M., and Patel, M. S.: The 
newborn of diabetic rat. 1. Hormonal and metabolic changes in the postnatal period. Pediatr. Res. 16: 632 (1982).

10. Girard, J. R., Caquet, D., Bal, D., and Guillet, I.: Control of rat liver phosphorylase. glucose-6-phosphatase and phosphoenolpyruvate carboxykinase activities by insulin and glucagon during the perinatal period. Enzyme, 15: 272 (1973).

11. Golob, E. K., Rishi, S., Becker, K. L., and Moore, C.: Streptozotocin diabetes in pregnant and nonpregnant rats. Metabolism. 19: 1014 (1970).

12. Gutmann. I. and Wahlefeld. A. W.: L-(+)-Lactate determination with Lactate Dehydrogenase and NAD. In: H. V. Bergmeyer: Methods of Enzlmatic Analisis. Vol. 3. pp. 1464-1468 (Academic Press, New York. 1974).

13. Hanson. R. W.. Reshet, L.. and Ballard. F. J.: Hormonal regulation of hepatic Penolpyruvate carboxykinase (GTP) during development. Fed. Proc., 34: 166 (1975).

14. Jaworek. D.. Gruber, W., Bergmeyer, H. V.: Adenosine-5'-disphosphate and Adenosine-5'-monophosphate. In: H. V. Bergmeyer: Methods of Enzymatic Analısis. Vol. 4. pp. 2127-2131 (Academic Press. New York, 1974).

15. Kalhan. S. C., Savin. S. M., and Adam. P. A. J.: Attenuated glucose production rate in newborn infants of insulin-dependent diabetic mothers. New Eng. J. Med.. 296: 375 (1977).

16. Lamprecht. W. and Trautschold, 1.: Adenosine-5'-Triphosphate. Determination with hexokinase and glucose-6-phosphate dehydrogenase. In: H. V. Bergmeyer: Methods of Enzimatic Analssis. Vol, 4. pp. 2101-2110 (Academic Press, New York, 1974).

17. Pedersen. J. and Osler. M.: Hyperglycemia as the cause of characteristic features of the foetus. Danish Med. Bull., $8: 78$ (1961)

18. Steele. J. G.. McGrath, M. C.. Yeoh, G. C. T.. and Oliver, I. T.: Phosphoenolpyruvate carboxykinase in cultured foetal hepatocytes from the rat. Ontogeny of hormone inducibility and role of glucocorticoids and insulin in enzyme

Copyright (C) 1982 International Pediatric Research Foundation, Inc. $0031-3998 / 82 / 1608-0638 \$ 02.00 / 0$ induction. Eur. J. Biochem.. 104: 91 ( 1980 ).

19. Sutton. R and Pollak. J. K. Hormone-initiated maturation of rat liver mitochondria after birth. Biochem. J.. 186: 361 (1980).

20. Van Handel. E.: Estimation of glycogen in small amounts of tissue. Anal Biochem.. II: 256 (1965).

21. Vernon, R. G. and Walker, D. G.: Gluconeogenesis from lactate in the developing rat. Studies in vivo. Biochem. J.. 127: 531 (1972).

22. Yeung. D. and Oliver, I. T.: Induction of phosphopyruvate carboxylase in neonatal rat liver by adenosine $3^{\prime} .5^{\prime}$-cyclic monophosphate. Biochemistry. 7 : 3231 (1968).

23. Williamson, D. H., Lund, P., and Krebs, H. A.: The redox state of free nicotinamide-adenine dinucleotide in the cytoplasm and mitochondria of rat liver. Biochem. J., 103: 514 (1967).

24. Wollenberger. A., Ristau, O., and Schoffa. G.: Eine einfache Technik der extrem schnellen Abkühlung großerer Gewebestucke. Pflugers Archiv., 270: 399 (1960).

25. Charles River Breeding Laboratories. Wilmington, MA. U.S.A.

26. J. M. Cuezva was a postdoctoral fellow of the Spanish Ministry of Universities and Research. His present address is the Department of Bioquimica and Biologia Molecular. Universidad Autonoma de Madrid, Madrid-34, Spain.

27. The present address of Dr. C. Chitra is Boston Biomedical Research Institute, 20 Staniford Street, Boston, MA. U.S.A

28. Requests for reprints should be addressed to: Dr. M. S. Patel, Department of Biochemistry. Case Western Reserve University School of Medicine, Cleveland, OH 44106 (U.S.A.).

29. This research was supported by Public Health Service Grants HD 11621 and HD 12643.

30. Received for publication August 19, 1981.

31. Accepted for publication January 13, 1982

Printed in U.S.A 